\title{
A Microphotometric Method for the Estimation of Penicillinase in Single Bacteria
}

\author{
By J. F. COLLINS, D. B. MASON AND W. J. PERKINS \\ National Institute for Medical Research, The Ridgeway, \\ Mill Hill, London, N.W. 7
}

(Received 8 May 1963)

\begin{abstract}
SUMMARY
A microphotometric technique is described by which the penicillinase content of a single bacterium in a stable microdrop is measured. The microdrop contains a buffer, benzylpenicillin and bromocresol purple. As the penicillinase hydrolyses the penicillin to penicilloic acid, the concentration of the cationic (purple) form of bromocresol purple decreases. This decrease is measured by a specially designed single-beam microphotometer, and, from the change in light transmission and the volume of the drop, the total penicillinase content of the drop is calculated. The microphotometer and the calibration of the assay with soluble penicillinase preparations are described.
\end{abstract}

\section{INTRODUCTION}

The specific enzyme activity of a bacterial culture is often of value in describing the state of the bacteria under experimental conditions, but the variation in enzyme content between the individual bacteria in the population has not been directly investigated before. The estimation of an enzyme in a single organism, in which only a few enzyme molecules may be present, requires novel assay techniques. A microphotometric technique has been developed for the assay of such quantities of penicillinase, and this technique may also be of general application to other enzyme assays. The basis of the technique is the observation of the enzymic reaction in a stable microdrop, which can contain either a bacterium whose penicillinase is to be estimated or a known concentration of soluble penicillinase for calibration purposes. Penicillinase catalyses the hydrolysis of penicillin to penicilloic acid, which has a secondary amino group and a carboxyl group that were not present in the original penicillin. At neutral $\mathrm{pH}$ values, the amino group is unionized but the new carboxyl group ionizes, and a proton is released. The solution thus tends to become acidic as the reaction proceeds, and in the assay system this proton release decreases the concentration of the ionized form and increases the concentration of the unionized form of a $\mathrm{pH}$ indicator, bromocresol purple, present in the assay mixture. The decrease in concentration of the purple (ionized form) is accompanied by an increasing light transmission through the solution, which is most marked at $5900 \AA$, the peak absorption of the purple form of the indicator. The optical measurements which enable the rate of the enzymic reaction in the microdrop to be calculated are made with the aid of a specially designed microphotometer, by which the transmission of the drop at a selected wavelength can be measured and recorded continuously. Since the observations are made of the transmission through a spherical

Vol. 34, No. 2 was issued 7 May 1964 
drop with an uncertain light path instead of the more usual parallel-sided container, the interpretation of the measurements is more complicated than in normal photometry, and a modified equation has been derived from Beer's law in which the concentration of the absorbing substance is introduced, but from which the light path and the extinction coefficient have been eliminated. Under the assay conditions used, this concentration can be assumed with only small errors.

The special microphotometer designed for the micro-assay is a single-beam instrument by which the light transmitted through a circular area about $1 \mu$ diameter in the centre of the drop under observation is detected by an 11-stage 'venetian blind' photomultiplier, and the output signal amplified and recorded on a potentiometric recorder. The instrument is designed to give high sensitivity together with stability over considerable periods of time.

The calibration of the method has shown the response to be linear over a considerable range of enzyme concentration, and the sensitivity is high enough to detect penicillinase activity corresponding to less than 50 molecules of Bacillus cereus penicillinase (Kogut, Pollock \& Tridgell, 1956).

\section{METHODS}

Reaction mixture. The usual reaction mixture used for the estimation of penicillinase contains $2.0 \mathrm{ml} .4 \%(\mathrm{w} / \mathrm{v})$ bromocresol purple (G. T. Gurr Ltd.; adjusted to $\mathrm{pH} 6 \cdot 15$ with sodium hydroxide), $1.0 \mathrm{ml} \cdot 0 \cdot 2 \mathrm{M}-\mathrm{K}_{2} \mathrm{HPO}_{4}, 0 \cdot 4 \mathrm{ml}$. benzylpenicillin solution (containing $2 \times 10^{5} \mathrm{i} . \mathrm{u} . / \mathrm{ml}$. saline) and $0.2 \mathrm{ml}$. saline. This mixture is at about $\mathrm{pH} \mathrm{7 \cdot 4}$. To make a drop preparation, a small quantity $(0.02 \mathrm{ml}$. $)$ of the test solution is mixed with $0.18 \mathrm{ml}$. of the reaction mixture. The test solution containing the penicillinase should have no strong buffering capacity.

Preparation of drops. Water-repellent tubes (treated with a solution of dimethyldichlorosilane: 'Repelcote', Hopkin and Williams Ltd.) were used for mixing the reaction mixture and the test solution. After vigorous mechanical mixing, the mixture was pipetted into a prepared water-repellent capillary tube which had been drawn out to a fine point on an automatic 'electrode' pulling machine (Alexander \& Nastuk, 1953). From the capillary tube, the mixture was sprayed into a drop of silicone oil (Midland Silicones Ltd., MS 702) on a water-repellent coverslip. By the use of a shield, drops were prevented from falling on to the surface of the coverslip itself. A second water-repellent coverslip was placed on top of the oil, and the oil and the drops trapped in it spread out between the two coverslips. The drops were just lighter or heavier than the oil, depending on the reaction mixture used, and came to rest touching one of the coverslips, but not wetting it, and retaining their spherical shape. Once the oil ceased flowing under the coverslip, the drops were stationary and stable for very long periods (i.e. days).

The microphotometer: the optical system. A restricted beam of light was used to illuminate the drop under observation on the stage of the microscope, and the light transmitted through the central area of the drop was measured with a photomultiplier. A schematic diagram is shown in Fig. 1. A Watson 'Bactil-60' microscope was used with a $\times 40$ air objective and a second matching objective in place of the normal substage condenser. The light source $A$, collector lens $B$, filter $C$ and field aperture $D$ were mounted on an optical bench. The light source was a $6 \mathrm{~V}$. 
$30 \mathrm{~W}$. bulb $20 \mathrm{in}$. from the substage condenser; the collector lens was $2 \frac{1}{4}$ in. from the bulb, so that the filament was focused at the substage condenser, giving Köhler illumination. The interference filter (max. transmission $5900 \AA, 74 \%$ transmission, band width $80 \AA$; Barr and Stroud Ltd.) was mounted in a swinging holder between the collector and the field aperture. The field aperture, $10 \mathrm{in}$. from the substage condenser, consisted of a demountable circular plate in a fixed holder, the aperture $(0.4 \mathrm{~mm}$. diam.) being drilled centrally in this plate. The mirror $(\boldsymbol{F})$ deflecting the light to the substage condenser was surface-silvered, to avoid multiple images of

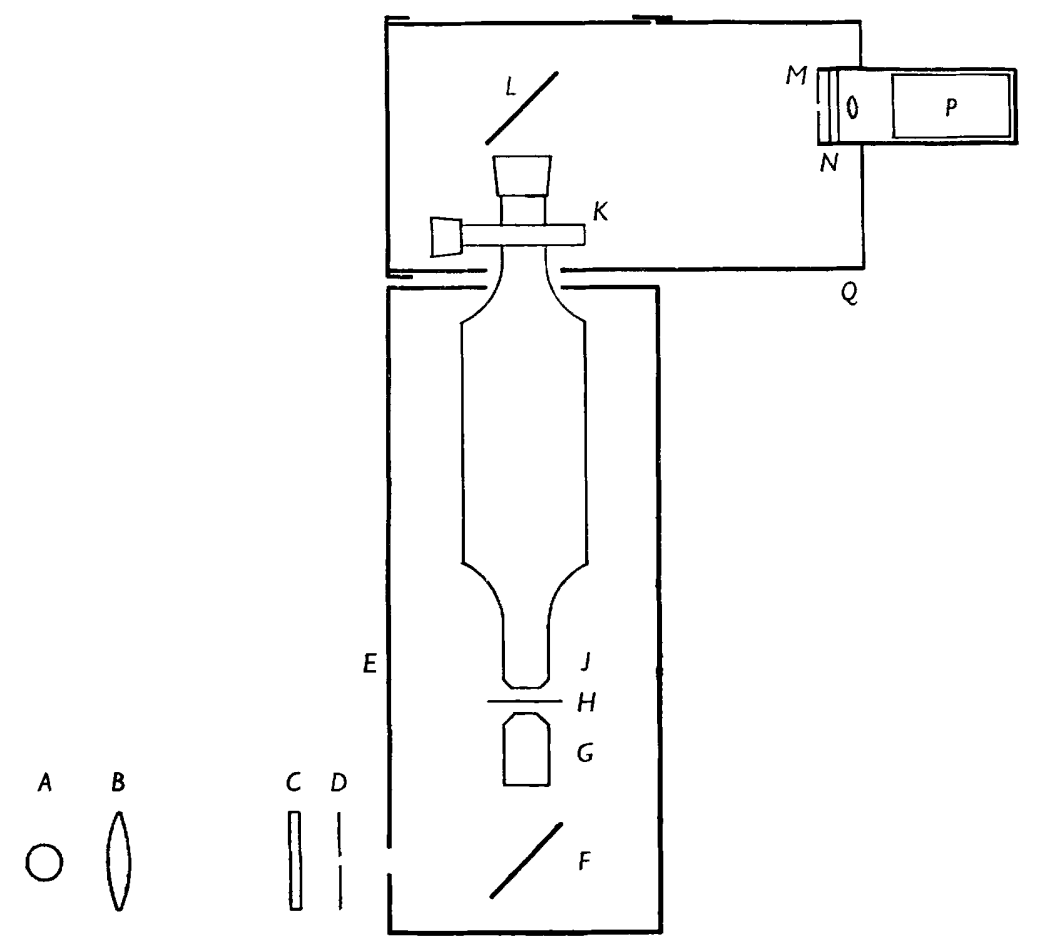

Fig. 1. Schematic diagram of microphotometer. $A$, lamp; $B$, condenser $C$, filter $\boldsymbol{D}$, field aperture; $E$, thermal enclosure; $F$, mirror ; $G, J, \times 40$ objectives; $H$, drop preparation; $K$, micrometer eyepiece; $L$, mirror ; $M$, screen with central aperture; $N$, camera shutter; $P$, photomultiplier; $Q$, light-tight enclosure.

the field aperture. The substage condenser $(G)$ was identical with the objective $(J)$, a $4 \mathrm{~mm} .(\times 40)$ air objective, N.A. 0.70. It was adjusted for each drop observed so that the image of the field aperture was in the same plane as the drop. A Baker $\times 10$ micrometer eyepiece $(K)$ was used, over which could be fitted a surface-silvered mirror $(L)$ deflecting the light through $90^{\circ}$. About $10 \mathrm{in}$. away the image of the drop was focused on a white screen $(M)$, and moved till it lay centrally about a $1 \mathrm{~mm}$. diam. hole, through which the light passed to the photomultiplier $(P)$. A camera shutter $(N)$ and a small lens to diverge the light beam over the face of the photomultiplier were mounted behind the sereen in a light-tight enclosure $(Q)$.

The microphotometer: electronic system. A single-beam instrument was chosen for overall simplicity, and adequate stability of the light source was obtained by using 
a 6 V. 30 W. bulb (F/25; G.E.C. Ltd.) fed from a 6 V. 7 A. transistor-stabilized d.c. power supply having an output impedance less than $0.01 \Omega$ and a stabilization factor for variations of input voltage greater than $5 \times 10^{3}$. The photomultiplier was an EMI type 6097 with an overall gain of the order of $10^{7}$. The EHT supply, which had a stabilization factor $>\mathbf{5 0 0}$ for variations of input voltage, provided $1250 \mathrm{~V}$. with a positive earth, and was applied between the cathode and last dynode of the photomultiplier, giving about $125 \mathrm{~V}$. across each dynode, the dynodes being joined by $330 \mathrm{k} \Omega$ resistors. The last dynode was earthed and the collector held at $+108 \mathrm{~V}$. with respect to it by the stabilizer valve $V_{2}$ (Fig. 2 ). The voltage developed across the collector load $\boldsymbol{R}_{L}$ by the current through the photomultiplier was applied to a balanced cathode follower amplifier (Fig. 2). A photomultiplier current of $0 \cdot 1 \mu \mathrm{A}$. when $R_{L}=1 \mathrm{M} \Omega$ provided an input to the amplifier of $100 \mathrm{mV}$., and this was arranged to give full-scale deflexion in the output. A balanced system was therefore

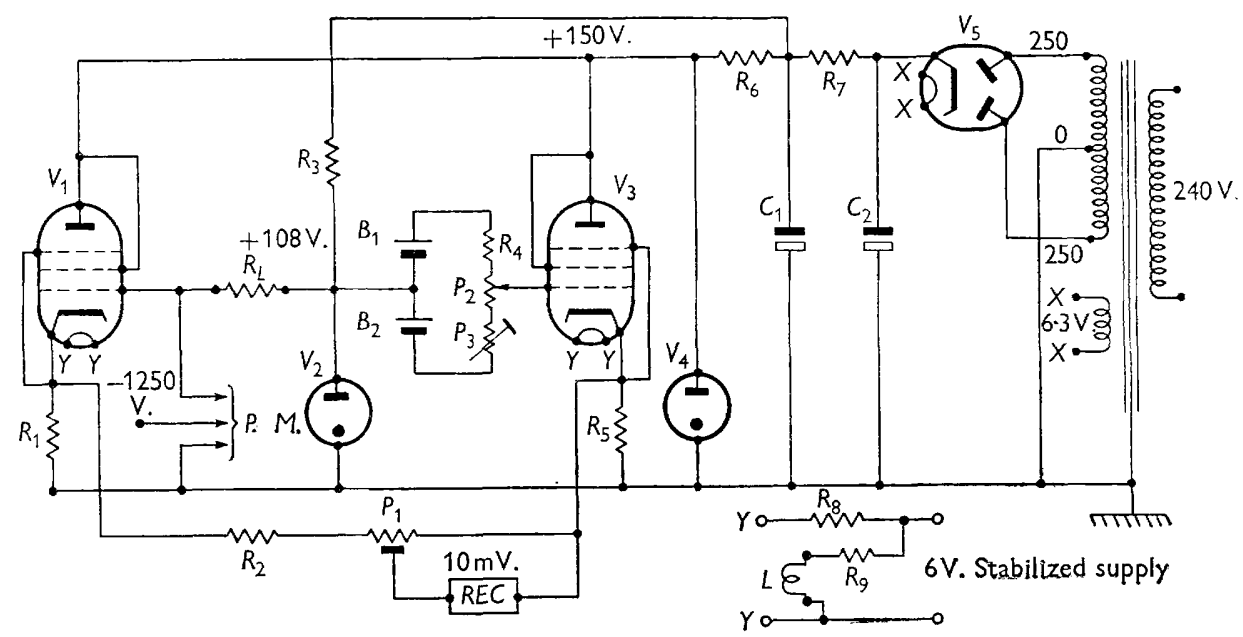

Fig. 2. Circuit of balanced cathode follower amplifier. $V_{1}, V_{3}, \mathrm{ME} 1400 ; V_{2}, \mathrm{M} 8224 ; V_{4}$, M 8223; $V_{5}, 6 \mathrm{X}_{4} ; \boldsymbol{R}_{1}, \boldsymbol{R}_{5}, 220 \mathrm{k} \Omega, 1 \% ; \boldsymbol{R}_{2}, 2 \cdot 7 \mathrm{k} \Omega ; \boldsymbol{R}_{3}, 18 \mathrm{k} \Omega ; \boldsymbol{R}_{4}, 68 \mathrm{k} \Omega ; \boldsymbol{R}_{6}, 12 \mathrm{k} \Omega ; R_{7}$, $1 \mathrm{k} \Omega ; \boldsymbol{R}_{8}, 5.5 \Omega ; \boldsymbol{R}_{9}, 0.5 \Omega, 120 \mathrm{~W} . ; \boldsymbol{R}_{L}, 1 \mathrm{M} \Omega, 500,250,150,100,50,33$ or $10 \mathrm{k} \Omega ; \boldsymbol{P}_{1}$, $500 \Omega$ potentiometer; $P_{2}, 10 \mathrm{k} \Omega$ potentiometer; $P_{3}, 100 \mathrm{k} \Omega$ potentiometer; $C_{1}, C_{2}$, $32 \mu \mathrm{F} . ; B_{1}, B_{2}$, RM-12R 1.35 V. batteries; P.M., EMI 6097 photomultiplier; $L, 6$ V. $30 \mathrm{~W} . \mathrm{F} / 25$ lamp; $R E C, 10 \mathrm{mV}$. servorecorder. Eight position selector switch for $\boldsymbol{R}_{L}$ omitted from diagram.

necessary to ensure that variations in the reference voltage were cancelled out. The cathode follower also provided a low output impedance from an input circuit of high impedance, allowing a conventional $10 \mathrm{mV}$. servorecorder to be used.

A pair of matched ME 1400 electrometer valves were used operating at $50 \mathrm{~V} . V_{a}$ (anode voltage), $0.5 \mathrm{~mA} . I_{a}$ (anode current) and $4.5 \mathrm{~V} . V_{f}$ (heater voltage), fed from the low voltage stabilized supply. Under these conditions grid currents were of the order of $10^{-12} \mathrm{~A}$. A series resistor in the output circuit dropped the $100 \mathrm{mV}$. to the $10 \mathrm{mV}$. required for the recorder and limited the output current to approximately $30 \mu \mathrm{A}$., which was small compared to the mean current through each valve $(500 \mu \mathrm{A})$. The potentiometer $\boldsymbol{P}_{2}$ enabled the amplifier to be adjusted to give zero output on the recorder when the photomultiplier collector was disconnected. The dark current 
from the photomultiplier was approximately $10^{-3} \mu \mathrm{A}$. at room temperature. To obtain a wide sensitivity range, eight collector loads covering a range of 100:1 could be selected.

The microphotometer: mechanical system. The apparatus was mounted on an integral platform supported by four antivibration mountings. The microscope was fixed to a plate which could be moved over three levelled studs, and locked to the platform. The optical bench was joined to a crossbar which was located at both ends against stops on the board. The microscope was lined up optically with source and aperture on the optical bench and then locked in position.

All the mechanical movement of the microscope stage was dismantled because it gave rise to slight movement of the stage after a drop had been centred. The moving slides were lubricated with a molybdenum disulphide suspension in grease, which enabled the stage to be manipulated satisfactorily, and precise centring of a drop was readily achieved.

A thermal jacket made of sheets of expanded polystyrene was built round the microscope, with a hole to allow the light to reach the instrument, and a removable side to allow adjustment of the stage and other controls. The thermal enclosure contained a heating coil and tank through which water at $30^{\circ}$ was continuously circulated. The temperature in the enclosure was measured near the stage, and with the side of the box closed the temperature rose rapidly to between 22 and $25^{\circ}$, depending on the ambient temperature. The eyepiece was enclosed in a light-tight tube leading to the photomultiplier; the end of this tube could be removed when direct observation either of the screen or through the eyepiece was required.

operation of the microphotometer. The electrical circuits were switched on about 30-60 min. before the photometer was required. When an enzyme assay was made, the drop preparation was made as described. It was then placed on the stage of the microscope, and the mirror on the eyepiece removed. The condenser was lowered, the field aperture removed and a neutral filter swung into the illuminating beam. The drops were then inspected carefully to find one which contained only a single organism. The diameter of the selected drop (typically $7-10 \mu$ ) was measured by means of the micrometer eyepiece. The field aperture was replaced, and the condenser racked up till the image of the field aperture appeared in the plane of focus of the drop. The mirror was then mounted on the eyepiece, and the field aperture again removed. An orange filter was introduced in place of the neutral filter, and adjustment of the fine focusing produced an image of the microscopic field on the screen $\boldsymbol{M}$ (Fig. 1). The microscope stage was then moved manually until the image of the selected drop was near, but not over, the aperture in the middle of the screen. The field aperture was replaced, and its image centred over the aperture in the screen $\boldsymbol{M}$ by means of the condenser centring screws. The light-tight cover between the microscope and the photomultiplier was closed, and the orange filter replaced by the interference filter. The camera shutter behind the screen $\boldsymbol{M}$ was opened, and the total light recorded on a low-sensitivity range of the photometer. After a short period to record this, the shutter was closed, the field aperture removed and the orange filter re-introduced in place of the interference filter. The end cap was taken off the tube between the microscope and the photomultiplier, and the microscope stage adjusted to bring the image of the drop centrally round the aperture in the middle of the screen $M$. The cap was replaced, the field aperture re-inserted and the 
interference filter replaced in the light path. The shutter was opened, and the light passing through the drop produced a signal which was recorded by the servorecorder. The collector load was chosen to give a reading about $25 \%$ of full scale on the recorder, which was then allowed to run for about 30-45 min., that is, until a stable temperature was reached inside the thermal jacket $E$, and a steady recording of the light signal obtained, from which the slope could be measured. The dark current was checked at intervals by interrupting the light path. It was also possible to check the response and stability of the system by introducing a fixed load across the input to the cathode follower, giving a steady signal.

\section{Calculation of enzyme activity in a drop}

Under the conditions of assay used, the rate of destruction of penicillin by the enzyme should be proportional to the amount of enzyme present, and for the initial period of the reaction the amount of penicillin hydrolysed changes a proportional amount of purple (cationic) bromocresol purple into the yellow form. Thus, if $E=$ total enzyme in drop, $c=$ concentration of purple bromocresol purple, $V=$ volume of the drop and $A$ is a constant:

$$
E=A V \frac{\mathrm{d} c}{\mathrm{~d} t}
$$

Now, Beer's law states that

$$
I=I_{0} \mathrm{e}^{-\alpha c d}
$$

where $I=$ transmitted light, $I_{0}=$ incident light, $\alpha=$ extinction coefficient, $c=$ the concentration of the absorbing material, and $d=$ the light path. If $I$ and $c$ are the only variables, differentiation of (2) with respect to time gives

$$
\begin{aligned}
\frac{\mathrm{d} I}{\mathrm{~d} t} & =-\alpha d I_{0} \mathrm{e}^{-\alpha_{c d}} \frac{\mathrm{d} c}{\mathrm{~d} t} \\
& =-\alpha d I \frac{\mathrm{d} c}{\mathrm{~d} t} .
\end{aligned}
$$

While the extinction coefficient and the light path may be considered to be known quantities, both of them could be open to question under the present circumstances because of: (1) possible changes in extinction coefficient under the high concentration conditions of the indicator, or changes caused by interaction with cell material; (2) the variable light path through the drop, which could be as long as the diameter of the drop, but which could be considerably shorter for light rays not passing through the exact centre of the drop. These two factors were therefore eliminated from the calculation in the following way. Taking logs of equation (2), and rearranging the terms

or

$$
\begin{gathered}
\log _{e} \frac{I_{0}}{I}=\alpha c d, \\
\frac{1}{c} \log _{e} \frac{I_{0}}{I}=\alpha d,
\end{gathered}
$$

$I$ and $I_{0}$ are known, and $c$ can be assumed to be the initial concentration $\left(C_{0}\right)$ of the purple form of bromocresol purple, if the readings are made within about $1 \mathrm{hr}$ of 
the start of the reaction (for the basal enzyme), by which time about $15 \%$ of the purple bromocresol purple has been converted (on average) into the yellow form. The final equation used for the calculation of the total enzyme present in the drop was obtained from equations (1), (3) and (4):

$$
E=-A V \frac{C_{0}}{I} \frac{1}{\log _{e} I_{0} / I} \frac{\mathrm{d} I}{\mathrm{~d} t} .
$$

The slope of the recording $(\mathrm{d} I / \mathrm{d} t)$ was measured about $30 \mathrm{~min}$. after the reaction started, but the recording was allowed to run on so that any gradual change in drop position which produced a gradual change in transmission would be more readily noticed. The temperature of the thermal enclosure was also noted, and the reaction rates were adjusted to the equivalent ones under the standard assay temperature of $30^{\circ}$.

\section{RESULTS}

\section{Stability and calibration of microphotometer}

Since the microphotometer was a single-beam machine, it was necessary to obtain a high degree of stability in the light source and the electronic equipment. The lamp (and valve heater) supply from the d.c. power supply and the EHT supply were held within close limits, and the stability of the whole system was tested by recording

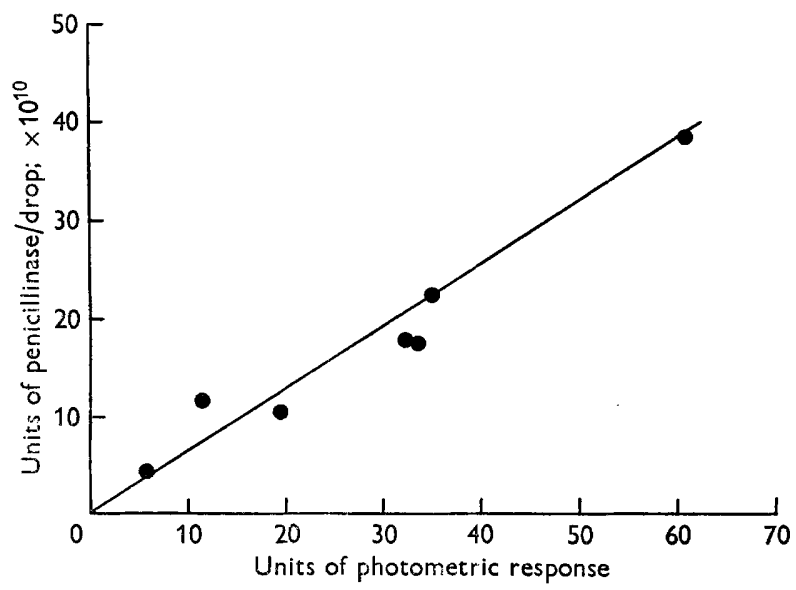

Fig. 3. Calibration curve of photometer. The relation between the soluble penicillinase content and the photometric response of microdrops.

the output from the photomultiplier illuminated through the optical system without a drop in the field. The recording showed no change over 3-4 hr, and this was accepted as a measure of the stability of the entire system, both optical and electrical.

The recording of the photomultiplier output signal was used to show that the components of the assay system were stable under the conditions of illumination used. Drop preparations containing only bromocresol purple indicator were rapidly bleached on illumination with white light, but the monochromatic light (wavelength $5900 \AA$ ) obtained from the interference filter caused no detectable change in light transmission for a period of $4 \mathrm{hr}$. Similar results were obtained when penicillin at 
the normal concentration was added to drops. Drops prepared from the bromocresol purple solution plus washed organisms showed a very slow change in transmission comparable to the least change produced by uninduced Bacillus lichenformis strain 749 organisms in the presence of the complete assay mixture. The contribution to the change in light transmission by the residual internal metabolism of one organism could be ignored under these circumstances, and no large error was introduced by attributing the entire change observed to penicillinase action.

Calibration. The microphotometer was calibrated by recording the transmission of drops of assay mixture in which had been mixed known concentrations of the soluble penicillinase released by Bacillus licheniformis organisms into the culture medium. Cell-bound penicillinase appeared to be readily accessible to the substrate, since the release of cell-bound penicillinase was not accompanied by an increase in the total enzyme activity measurable; hence the calibration with soluble penicillinase could be validly applied to the results obtained for cell-bound enzyme. The results of the calibration experiments are shown in Fig. 3. A linear relation between the amount of penicillinase present in the drop and the photometric response was obtained.

\section{DISCUSSION}

The technique and the microphotometer. The development of the technique for the assay of small amounts of enzymes in microdrops has enabled investigations of the variation within a bacterial population to be undertaken (Collins, 1964). This technique uses basically the photometric method common to many ordinary enzyme assays. In the microdrop the progress of the reaction caused by the enzyme is observed at a selected wavelength, and the continuous recording of the reaction allows the calculation of the rate of enzymic reaction per unit volume of the drop. The volume of the drop is estimated from its visible diameter, assuming it to be spherical. This assumption was justified, since a moving drop coming to rest against the coverslip underwent no visible change of appearance. The moving drop is practically spherical, and on touching the coverslip there will be a contact pressure equal to the difference in weight between the drop and the oil it displaces. This contact pressure, transmitted through the drop, causes the horizontal (visible) diameter of the drop to increase slightly and the vertical diameter to decrease slightly, but the changes in diameter were calculated theoretically to be of the order of $0.1 \%$ of the diameter of the sphere, and were disregarded. The stability of drops in the preparations in oil, between water-repellent coverslips, enabled the method to be used successfully.

The light path through the drop was eliminated from the calculation of the rate of reaction, but the transmitted light through the drop has an added component of light diffracted round the edge of the drop. Trials with slides covered with droplets of mercury (evaporated on to the slide) in oil showed that this diffracted light could be as much as $20 \%$ of the light transmitted through a bromocresol purple drop. However, in the drop preparations the intensity of the diffracted light is decreased from that in the model system, since the refractive index change at the oil-drop interface is much smaller. It is difficult to estimate the diffracted light for the drop preparations, but from the good calibration figures it is unlikely the contribution to the transmitted light could be more than $10 \%$ of the light signal for an average 
sized drop. The fact that the light path has been eliminated from the calculations means that the method is not restricted to spherical drops, but can be used to assay enzyme in any stable preparation whose volume is known.

The single-beam microphotometer has been successfully used in the measurements of light transmitted through drops, because of the high stability that has been achieved in the electronic and mechanical components. The manner in which this stability has been achieved has already been indicated in Methods. It may be added that the apparatus was operated in a laboratory containing the usual quota of centrifuges, etc.

The stability of the equipment was shown in the series of control experiments that were made with actual drop preparations. The use of soluble enzyme preparations enabled calibration of the response of the microphotometer over a wide range of enzyme concentrations; the results (Fig. 3) gave good agreement with the linear response expected. The errors involved in the calculation have been assessed roughly as $\pm 5 \%$ for $C_{0}, \pm 2 \frac{1}{2} \%$ for the diameter (equivalent to $\pm 7 \frac{1}{2} \%$ for the volume $V$ ), $\pm 10 \%$ for the total and transmitted light and the rate of change of light transmission. These give an estimated error of about $\pm \mathbf{3 5} \%$ in the calculated values of total enzyme in the drop. The calibration figures lie mostly within these limits, though a few larger deviations were also found.

The assay mixture. The assay mixture was so designed that, when all the penicillin had been destroyed, the $\mathrm{pH}$ value should be approximately $6 \cdot 15$, the $\mathrm{pK}$ value of bromocresol purple. The concentration of the purple form of the indicator was initially practically the same as the total concentration of the indicator itself, and the final concentration should be half this; in this way the concentration of the purple form needed for the calculations could be assumed without gross error to be that initially present, and the error should always be in the same sense. During the initial period of the enzymic reaction, there is competition between the purple form of the indicator and phosphate ions for the protons being released, and the change in concentration of the purple form of the indicator should bear a linear relation to the amount of penicillin destroyed. Thus the assumption of a linear relation between photometric units and the amount of enzyme present in the drop is reasonable.

Application to bacteria. The analysis of the distribution of penicillinase in bacterial populations as determined by using the technique described here is given in the next paper (Collins, 1964). Changes in most drops containing single organisms of Bacillus licheniformis were readily observed. It was noted on occasion that, after a drop preparation had been left overnight, drops containing organisms had changed colour, but drops without organisms remained purple.

The general method. The method should be applicable in principle to the assay of an enzyme which can be made to produce a measurable change in the light transmission of a microdrop. There are, however, other requirements for the successful operation of the method, which have not been stressed so far. It is particularly important that the enzyme substrate and product should be insoluble in oil so that they do not leave the microdrop during the observations. The indicator or chromogen used must also be insoluble in oil and stable to the lighting conditions used. In the case of penicillinase, these requirements have been easily met, and the high molecular activity of the penicillinase used has enabled the activities of 50 molecules 
of it to be detected. It may not be possible to reach such sensitivity with other enzymes. Since relatively few enzyme molecules are present in a microdrop, it may be necessary to guard against enzyme inactivation at the oil/water interface, by the addition of carrier protein. In the case of penicillinase this was not necessary.

The viability of the organisms after they had been sprayed into the microdrops for penicillinase assays was not tested. To retrieve such an organism would require very careful micromanipulation, and was not attempted because the high concentration of penicillin in the assay mixture made survival rather unlikely. The reverse operation of inserting an organism into a drop may be simpler and more useful in practice.

We wish to thank Dr M. R. Pollock, F.R.S., for his interest and encouragement during the development of this technique, Mr J. Smiles, O.B.E., and Mr M. R. Young for valuable advice, and Mr W. Perrin for building the amplifier.

\section{REFERENCES}

Alexander, J. T. \& Nastuk, W. L. (1953). An instrument for the production of microelectrodes used in electrophysiological studies. Rev. sci. Instrum. 24, 528.

Collins, J. F. (1964). The distribution and formation of penicillinase in a bacterial population of Bacillus licheniformis. J. gen. Microbiol. 34, 363.

Kogut, M., Pollock, M. R. \& Tridgels, E. J. (1956). Purification of penicillin-induced penicillinase of Bacillus cereus NRRL 569: a comparison of its properties with those of a similarly purified penicillinase produced spontaneously by a constitutive mutant strain. Biochem. J. 62, 391. 